\title{
ALTERNATIVAS DE ANÁLISE DE ENSAIOS EM LÁTICE NO MELHORAMENTO VEGETAL ${ }^{1}$
}

\author{
JÚLIO SÍLVIO DE SOUSA BUENO FILHO² e ROLAND VENCOVSKY³
}

\begin{abstract}
RESUMO - Compararam-se diferentes formas de análise de experimentos em blocos incompletos, abordadas como casos particulares de modelos mistos, quais sejam: (a) análise intrablocos, em que apenas o efeito do erro experimental é suposto aleatório; (b) análise interblocos (látice), com efeitos de blocos supostos aleatórios; (c) análise BLUP, com os efeitos de tratamentos supostos aleatórios, e (d) modelo aleatório. Além disso, montou-se a ANAVA, considerando duas alternativas: (e) usando o quadrado médio de tratamentos ajustados para blocos e o quadrado médio do erro efetivo do látice; (f) tomando as repetições como blocos completos. Um exemplo de análise de um teste de progênies de Eucalyptus grandis (Hill) Maiden ilustra as implicações da escolha dos modelos para fins de seleção e de caracterização genética de populações. Observou-se que em geral o ordenamento dos tratamentos sofreu maiores alterações ao se mudar a alternativa de análise do que as estimativas do progresso esperado pela seleção. Tendência que se reforça com a seleção mais intensa. As formas de análise que consideram a restrição da casualização (blocos incompletos) foram as mais precisas, e dentre estas, a análise BLUP de tratamentos é conceitualmente a melhor, pois os tratamentos eram progênies de polinização livre, sendo a que mais difere da análise usual do látice. Isto indica ser possível minorar os erros de seleção nas análises de blocos incompletos no melhoramento vegetal.
\end{abstract}

Termos para indexação: modelos lineares, delineamentos experimentais, blocos incompletos parcialmente balanceados, modelos mistos, BLUP.

\section{ALTERNATIVES OF LATTICE DESIGN ANALYSIS IN PLANT BREEDING}

ABSTRACT - A comparison was made between the alternative models of analysis in a mixed model approach to partially balanced incomplete block designs, namely: (a) fixed model; (b) analysis with recovery of interblock information (lattice); (c) BLUP analysis of treatment's effects; and (d) random model. Two alternatives for construct the ANOVA table was also considered: (e) to take the mean squares of treatments adjusted to blocks and lattice effective errors; (f) to take the replications as random complete blocks. An Eucalyptus grandis (Hill) Maiden progeny trial illustrates the implications of model choice in selection and variability studies. It was found that, in general, the ranking of treatment means was more affected by the type of model chosen than the estimation of genetic progress expected from selection. This was particularly true under higher selection intensities. The analysis forms that consider the restriction of the randomization (incomplete blocks) were the most precise and among them, the analysis BLUP of treatments is conceptually the best (because the treatments were open pollination progenies), being the one that differs most of the usual analysis of the lattice. This indicates to be possible to lessen the selection mistakes in the analyses of incomplete blocks in plant breeding.

Index terms: linear models, experimental designs, partially balanced incomplete block designs, mixed model methods, BLUP.

\footnotetext{
${ }^{1}$ Aceito para publicação em 5 de abril de 1999.

${ }^{2}$ Eng. Agrôn., Dr., Prof. Adjunto, Universidade Federal de Lavras (UFLA), Caixa Postal 37, CEP 37200-000 Lavras, MG. E-mail: jssbueno@ufla.br

${ }^{3}$ Eng. Agrôn., Dr., Prof. Titular, Dep. Genética, ESALQ/USP, Caixa Postal 83, CEP 13400-000 Piracicaba, SP.
}

\section{INTRODUÇÃO}

Os ensaios em látice foram primeiro introduzidos por Yates (1936), com vistas a permitir a análise de um grande número de tratamentos. Tais ensaios são bastante utilizados no melhoramento de plantas, seja para comparações entre variedades, na 
análise dialélica, ou para testes envolvendo dezenas a centenas de progênies na seleção recorrente.

Os látices são delineamentos em blocos incompletos com desbalanceamento planejado que permitem variadas formas de análise, das quais se destacam a análise intrablocos e a análise com recuperação da informação interblocos (Cochran \& Cox, 1976). Outro esquema usual é a análise como blocos ao acaso, utilizando-se as médias ajustadas de tratamentos e o erro efetivo oriundos da análise completa (interblocos) para montar somas de quadrados análogas às do delineamento em blocos completos. Tal análise foi primeiro adotada por Eberhart (1970).

Cecon (1992) estudou diferentes alternativas de análise dos látices, incluindo, além das já citadas, aquela que assume as repetições como blocos completos casualizados, o que também é usual, especialmente nos casos de erros no estabelecimento do arranjo experimental (Bueno Filho, 1992). A esse respeito, Miranda Filho (1987) sugeriu que a análise completa do látice somente traria vantagens sobre a análise em blocos ao acaso, tomando-se as repetições como blocos completos, se a sua eficiência fosse superior a $110 \%$.

Cecon (1992) concluiu que a melhor alternativa de análise para fins de estimativas de parâmetros genéticos é considerar sempre a casualização do látice e a recuperação da informação interblocos, qualquer que seja o valor da eficiência do látice. $\mathrm{O}$ autor apresenta as diferentes expressões para os componentes da variância, incluindo a variância entre médias ajustadas de tratamentos, cujos efeitos são fixos para fins de estimação no látice, não tendo componentes da variância a eles associados.

Deve-se notar que, mesmo em ensaios com tratamentos que possuem variabilidade intrínseca (por exemplo, de natureza genética) esta é uma prática usual. Como exemplo recente, Silva (1997) apresenta uma série de comparações entre quatro modelos de análise de látices, e discute, além dos já apresentados, a análise intrablocos com somas de quadrados de tratamentos ajustados para blocos. O autor obteve também as diferentes expressões algébricas para as esperanças dos quadrados médios, supondo que tratamentos e blocos estivessem representados por efeitos aleatórios. Ao encontrar altas correlações de Spearman entre as médias ordenadas em cada tipo de análise, o autor conclui que, quanto ao ordenamento, as análises intrablocos e interblocos praticamente não diferem.

No contexto de modelos lineares mistos, as diferentes alternativas de análise do látice que podem ser consideradas resumem-se a considerar apenas o erro experimental aleatório (análise intrablocos); efeitos aleatórios de blocos (recuperação da informação interblocos); efeitos aleatórios de tratamentos (best linear unbiased prediction - BLUP, para a predição de valores de progênies) e modelo aleatório (ou de componentes da variância); além da análise das repetições como blocos completos.

Segundo Robinson (1991), foram Henderson et al. (1959) os primeiros a demonstrar que as equações de modelos mistos produziam como soluções (BLUE dos tratamentos) os estimadores de recuperação da informação interblocos, além dos preditores (BLUP) dos efeitos aleatórios.

White \& Hodge (1989) recomendam que, para a predição de valores aditivos em testes de progênies florestais, os efeitos genéticos devem ser considerados aleatórios, e todos os demais efeitos, fixos. Tal abordagem corresponde, didaticamente, a uma compensação dos efeitos fixos de cada observação, seguida da análise do modelo aleatório residual. Nessa análise deve-se levar em conta a influência das componentes da variância na determinação dos preditores (estimadores) de efeitos aleatórios.

Bueno Filho (1997) encontrou diferenças de ordenamento em ensaios florestais decorrentes unicamente de se considerar o modelo como fixo ou aleatório no processo de predição. Isso ressalta a importância da decisão prévia sobre a natureza do modelo da análise, pois esta pode levar a erros de ordenamento (erros de seleção) e de predição de ganhos pela seleção, entre outros.

Este trabalho visa ilustrar algumas dessas alterações num ensaio em látice, e apontar, entre as alternativas de análise, as mais adequadas para o melhoramento vegetal.

\section{MATERIAL E MÉTODOS}

\section{Material vegetal}

O material vegetal utilizado consistiu de um teste de progênies de polinização livre (supostamente de meios- 
irmãos) de Eucalyptus grandis (Hill) Maiden, instalado como látice triplo triplicado no município de Salto, SP, no ano de 1979. As medidas de diâmetro à altura do peito (DAP, expresso em centímetros), altura de árvores (em metros), e volume em $\mathrm{dm}^{3}$, calculado segundo equação volumétrica apresentada em Bueno Filho (1992), foram tomadas aos sete anos, de árvores individuais, reunidas em parcelas de dez árvores. Os dados foram cedidos pela Duraflora S.A., empresa de reflorestamento com unidade experimental em Agudos, SP.

\section{Modelo da análise}

A análise seguiu o modelo estatístico geral: $Y_{i j k}=m+\tau_{i}+r_{j}+\beta_{k(j)}+e_{i j k}$, em que: $Y_{i j k}$ é o valor observado do tratamento i no bloco $\mathrm{k}$, dentro da repetição $\mathrm{j}$; $\mathrm{m}$ é a média geral do experimento; $\tau_{\mathrm{i}}$ é o efeito do tratamento $\mathrm{i}$, com i variando de $1 \mathrm{a} 100 ; \mathrm{r}_{\mathrm{j}}$ é o efeito da repetição, com j variando de 1 a $9 ; \beta_{\mathrm{k}(\mathrm{j})}$ é o efeito do bloco $\mathrm{k}$, hierarquizado dentro da repetição $\mathrm{j}$, com $\mathrm{k}$ variando de 1 a 10 na primeira repetição até 81 a 90 na última repetição; $\mathrm{e}_{\mathrm{ijk}}$ é o erro experimental associado ao total da parcela $\mathrm{Y}_{\mathrm{ijk}}$. Adotou-se a suposição de que os erros sejam normal e identicamente distribuídos, com média 0 e variância $\sigma_{\mathrm{e}}^{2}$. A mesma suposição de normalidade foi adotada para as variáveis aleatórias nos modelos abaixo.

\section{Formas de análise comparadas}

Foram empregadas, para a estimação, as seguintes formas de análise a ser comparadas:

(a) Análise intrablocos, tratamentos não ajustados (modelo fixo);

(b) Análise com recuperação da informação interblocos: efeitos fixos de tratamento e repetições e efeitos aleatórios de blocos dentro de repetições (modelo látice);

(c) Predição de valores aleatórios de tratamentos: efeitos aleatórios de tratamento e efeitos fixos de repetições e de blocos dentro de repetições (BLUP);

(d) Modelo de componentes da variância: modelo aleatório;

(e) Processo (b) para estimar os efeitos de tratamentos ajustados para blocos, seguido do processo (d) para estimar componentes da variância a partir da ANAVA;

(f) Modelo de blocos ao acaso, com cada repetição do experimento tratada como bloco completo.

\section{Estimação-predição}

O método geral de estimação partiu das equações de modelos mistos, das quais cada um dos casos (a) a (d) são particularidades. Os casos (e) e (f) levam em conta as es- peranças de quadrados médios do modelo aleatório de blocos casualizados completos (Vencovsky \& Barriga, 1992).

Seja o modelo $\mathrm{Y}=\mathrm{XB}+\mathrm{Zu}+\mathrm{e}$, em que $\mathrm{X}$ e $\mathrm{Z}$ são as matrizes de incidência de efeitos fixos e aleatórios, respectivamente, B é o vetor de efeitos fixos a serem estimados e u o vetor de efeitos aleatórios a serem preditos, com $\mathrm{E}(\mathrm{u})=0$ e $\mathrm{E}(\mathrm{e})=0$, e

$$
\operatorname{Var}\left(\begin{array}{l}
\mathrm{u} \\
\mathrm{e}
\end{array}\right)=\left[\begin{array}{cc}
\mathrm{G} & 0 \\
0 & \mathrm{R}
\end{array}\right] \sigma^{2} \text { (Henderson, 1975), }
$$

com $\sigma^{2}$ constante (variância residual) e, para cada análise, $\mathrm{R}=\mathrm{I}$, (matriz identidade). A matriz $\mathrm{G}$ não se define para o modelo fixo e é suposta conhecida, positiva-definida, para os modelos com efeitos aleatórios, como se segue:

(a) G não se define, confundindo-se com R;

(b) $G=D\left\{\sigma_{\beta}^{2} / \sigma^{2}\right\}$;

(c) $\mathrm{G}=\mathrm{D}\left\{\boldsymbol{\sigma}_{\tau}^{2} / \sigma^{2}\right\}$;

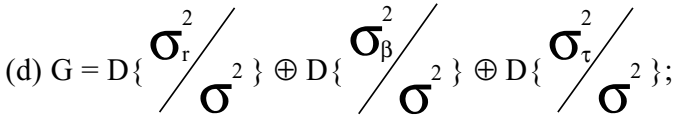

em que D representa a matriz diagonal dos elementos entre chaves, e $\oplus$ representa a soma direta de matrizes (resultando numa matriz diagonal não uniforme); $\sigma_{\beta}^{2}$, corresponde à variância de blocos; $\sigma_{\tau}^{2}$ corresponde à variância de tratamentos e $\sigma_{\mathrm{r}}^{2}$ corresponde à variância de repetições.

As equações de modelos mistos assumem a forma:

$$
\begin{aligned}
& {\left[\begin{array}{cc}
X^{\prime} R^{-1} X & X^{\prime} R^{-1} Z \\
Z^{\prime} R^{-1} X & Z^{\prime} R^{-1} Z+G^{-1}
\end{array}\right]\left(\begin{array}{l}
B \\
u
\end{array}\right)=\left(\begin{array}{c}
X^{\prime} R^{-1} y \\
Z^{\prime} R^{-1} y
\end{array}\right) \Rightarrow} \\
& {\left[\begin{array}{cc}
X^{\prime} X & X^{\prime} Z \\
Z^{\prime} X & Z^{\prime} Z+G^{-1}
\end{array}\right]\left(\begin{array}{l}
B \\
u
\end{array}\right)=\left(\begin{array}{l}
X^{\prime} y \\
Z^{\prime} y
\end{array}\right) \text { (Henderson et al., }} \\
& 1959) .
\end{aligned}
$$

Para a obtenção das soluções dessas equações, supõese uma solução inicial para G, ou seja, as relações entre as componentes da variância dos efeitos aleatórios e variância residual, exceto para o caso (a), em que se supõe não haver efeitos aleatórios além do erro experimental. A cada passo de iteração a estimação de uma nova matriz $\mathrm{G}$ permite melhorar tanto as predições quanto as estimativas de efeitos fixos e componentes da variância. 


\section{Forma de cálculo}

As estimativas das componentes da variância, bem como as soluções dos efeitos aleatórios, foram calculadas num procedimento único iterativo de estimação-predição, pelo método da Máxima Verossimilhança Restrita (REML), através da Proc MIXED do SAS, com estimativa inicial de G pelo método MIVQUE0 (Latour et al., 1994).

As estimativas de componentes da variância pelo método dos momentos no delineamento em blocos casualizados e modelo aleatório [casos (e) e (f)] foram obtidas utilizando a Proc GLM do SAS para as esperanças de quadrados médios tipo I, ou seja, pelo ajuste sucessivo de modelos, ou redução seqüencial (SAS Institute, 1988).

\section{Comparações}

Compararam-se as diferentes análises do ponto de vista das estimativas de componentes da variância com vistas a buscar possíveis erros de caracterização genética resultantes de se considerar efeitos fixos como aleatórios e viceversa. Calcularam-se também as herdabilidades no sentido restrito para a seleção com base nas médias de progênies. Para os modelos (a) e (b), a herdabilidade é dada pela expressão:

$$
\hat{\mathrm{h}}_{\mathrm{m}}^{2}=\frac{\text { QMProg. }- \text { QMErro }}{\text { QMProg. }},
$$

em que QMProg. e QMErro são, respectivamente, os quadrados médios de progênies e do erro no quadro de análise da variância. Para os modelos de componentes da variância, por outro lado, a herdabilidade é estimada por:

$$
\hat{\mathrm{h}}_{\mathrm{m}}^{2}=\frac{\hat{\sigma}_{\tau}^{2}}{\hat{\sigma}_{\tau}^{2}+\hat{\sigma}^{2} / \mathrm{r}},
$$

em que r é o número de repetições (9), $\hat{\sigma}_{\mathrm{t}}^{2}$ e $\hat{\sigma}^{2}$ são, respectivamente, as estimativas de máxima verossimilhança residual (REML) da variância de progênies e da variância do erro experimental.

Os intervalos exatos de $95 \%$ de confiança para o coeficiente de herdabilidade foram calculados como propostos por Knapp et al. (1985). Tal intervalo é obtido por meio da relação entre o coeficiente de herdabilidade e as razões entre os quadrados médios da ANAVA. Para o caso das análises (c) e (d), tais quadrados médios foram remontados a partir das estimativas REML das componentes da variância.

Compararam-se as seleções preditas nos diferentes ordenamentos tomando-se o modelo (b) como padrão (por ser o mais empregado), para a proporção de $2 \%$ a $50 \%$ de progênies selecionadas, por meio da expressão de Hamblin \& Zimmerman (1986) para a eficiência, qual seja:

$\mathrm{EF}(\mathrm{x} / \mathrm{b})=\frac{\mathrm{A}-\mathrm{C}}{\mathrm{B}-\mathrm{C}} \times 100 \%$,

em que $E F(x / b)$ é a eficiência de um ordenamento qualquer em relação ao modelo (b), A é o número de tratamentos cujo ordenamento foi coincidente; $\mathrm{B}$ é o número de tratamentos considerados e $\mathrm{C}$ é o número de coincidências casuais esperado em média para dado número de progênies selecionadas (estimado pelo produto entre a proporção das progênies selecionadas e o número de progênies selecionadas).

Essa mesma expressão foi utilizada para calcular a eficiência do modelo (f) em relação ao modelo (c), para a proporção de $2 \%$ a $50 \%$ de progênies selecionadas. Calcularam-se valores médios dessa eficiência para algumas faixas de intensidade de seleção, para maior clareza dos resultados.

Calcularam-se também os progressos genéticos esperados pela seleção de $2 \%$ a $35 \%$ das melhores progênies conforme cada modelo de estimação-predição.

\section{RESULTADOS E DISCUSSÃO}

As análises de variância para os diferentes modelos, para os três caracteres considerados, estão sumariadas na Tabela 1. Por essa tabela, nota-se que quaisquer dos diferentes tipos de análise acusariam diferenças entre os efeitos de progênies pelo teste $F$, a um nível de significância muito baixo. Tal fato indica a boa qualidade dos ensaios, bastante precisos em revelar diferenças entre os tratamentos, bem como a eficácia do controle local, que se nota na alta significância dos efeitos de blocos e na significância dos efeitos de repetições, embora essas incluíssem grandes áreas. Verificaram-se também eficiências dos látices sobre blocos ao acaso da ordem de $115 \%$ a $120 \%$, indicando não ser adequado ignorar a variação devida a blocos dentro de repetições. As estimativas da variância do resíduo foram semelhantes para todos os modelos de análise fundamentados no erro intrablocos, sendo bem maiores para a análise em blocos casualizados completos (modelo f). Com efeito, mesmo incluindo o modelo (e) que leva em conta o erro efetivo, os coeficientes de variação experimental das análises que levam em conta a presença de blocos incompletos foram ligeiramente menores que os da análise em blocos completos (em 
média, $10 \%$ versus $11 \%$ para o DAP, $7 \%$ versus $8 \%$ para a altura e $24 \%$ versus $26 \%$ para o volume).

O modelo aleatório foi o único que necessitou de mais de um passo de iteração na estimação REML, assim o grau de desbalanceamento dos dados pode ser considerado baixo, tanto para a recuperação da informação interblocos (efeitos aleatórios de blocos) quanto para a predição dos efeitos aleatórios de pro-

TABELA 1. Análise da variância para os seis modelos e três caracteres considerados. Teste de progênies E. grandis, Salto, 1979, aos sete anos de idade.

(a) Modelo: Fixo

\begin{tabular}{lrccc}
\hline Fonte de variação & GL & DAP $(\mathrm{cm} / \text { parcela })^{2}$ & Altura $(\mathrm{m} / \text { parcela })^{2}$ & ${\text { Volume }\left(\mathrm{dm}^{3} / \text { parcela }^{2}\right.}^{2}$ \\
\hline Repetições & 8 & $3773,9254^{* *}$ & $4302,3623^{* *}$ & $13984,8876^{* *}$ \\
Blocos(Rep. aj.) & 81 & $454,3245^{* *}$ & $1187,6343^{* *}$ & $2123,2356^{* *}$ \\
Tratamentos & 99 & $549,0395^{* *}$ & $1389,2757^{* *}$ & $2215,8639^{* *}$ \\
Resíduo (EIB) & 711 & 155,4705 & 294,6139 & 631,8053 \\
\hline
\end{tabular}

(b) Modelo: Látice

\begin{tabular}{|c|c|c|c|c|}
\hline Fonte de variação & GL & DAP $(\mathrm{cm} / \text { parcela })^{2}$ & Altura $(\mathrm{m} / \text { parcela })^{2}$ & Volume $\left(\mathrm{dm}^{3} / \text { parcela }\right)^{2}$ \\
\hline Tratamentos (aj.) & 99 & $551,6317^{* *}$ & $1431,2724 * *$ & $2229,1071 * *$ \\
\hline Resíduo (EIB) & 711 & 155,3892 & 294,5005 & 631,4715 \\
\hline
\end{tabular}

(c) Modelo: BLUP

\begin{tabular}{lrcc}
\hline Componentes da variância & DAP $(\mathrm{cm} / \text { parcela })^{2}$ & Altura $(\mathrm{m} / \text { parcela })^{2}$ & ${\text { Volume }\left(\mathrm{dm}^{3} / \text { parcela }\right)^{2}}^{\text {Tratamentos }}$ \\
Resíduo & $48,1656 \pm 9,6546$ & $134,3087 \pm 24,4232$ & $193,7053 \pm 38,9251$ \\
& $155,4897 \pm 8,2460$ & $294,6843 \pm 15,6306$ & $631,8340 \pm 33,5054$ \\
\hline
\end{tabular}

(d) Modelo: Aleatório

\begin{tabular}{|c|c|c|c|}
\hline Componentes da variância & $\mathrm{DAP}(\mathrm{cm} / \text { parcela })^{2}$ & Altura $(\mathrm{m} / \text { parcela })^{2}$ & Volume $\left(\mathrm{dm}^{3} / \text { parcela }\right)^{2}$ \\
\hline Repetições & $33,3875 \pm 18,8831$ & $32,7993 \pm 21,5753$ & $119,5086 \pm 70,0020$ \\
\hline Blocos(Rep.) & $27,9330 \pm 7,0915$ & $72,8094 \pm 16,6160$ & $140,1086 \pm 32,9725$ \\
\hline Tratamentos & $46,6097 \pm 9,3030$ & $135,4277 \pm 24,3586$ & $188,9268 \pm 37,7826$ \\
\hline Resíduo & $155,8843 \pm 8,2714$ & $294,3443 \pm 15,5785$ & $633,0030 \pm 33,5743$ \\
\hline
\end{tabular}

(e) Modelo: $\mathrm{BC}$ - Tratamentos ajustados para blocos e erro efetivo do látice

\begin{tabular}{lcccc}
\hline Fonte de variação & GL & DAP $(\mathrm{cm} / \text { parcela })^{2}$ & Altura $(\mathrm{m} / \text { parcela })^{2}$ & ${\text { Volume }\left(\mathrm{dm}^{3} / \text { parcela }^{2}\right.}^{2}$ \\
\hline Tratamentos (aj.) & 99 & $551,6317^{* *}$ & $1431,2724^{* *}$ & $2229,1071^{* *}$ \\
Erro efetivo & 711 & 157,6713 & 299,8869 & 642,4198 \\
\hline
\end{tabular}

(f) Modelo: Análise tomando repetições como blocos completos

\begin{tabular}{lrccc}
\hline Fonte de variação & GL & DAP $(\mathrm{cm} / \text { parcela })^{2}$ & ${\text { Altura }(\mathrm{m} / \text { parcela })^{2}}^{2}$ & ${\text { Volume }\left(\mathrm{dm}^{3} / \text { parcela }^{2}\right.}^{2}$ \\
\hline Repetições & 8 & $3773,9254^{* *}$ & $4302,3623^{* *}$ & $13984,8876^{* *}$ \\
Tratamentos & 99 & $579,5512^{* *}$ & $1592,5620^{* *}$ & $2375,0460^{* *}$ \\
Resíduo & 792 & 182,2211 & 360,5347 & 764,4402 \\
\hline
\end{tabular}

Eficiência do látice (b) com relação ao delineamento em blocos ao acaso (f) e média geral dos ensaios

\begin{tabular}{lccc}
\hline Parâmetro & DAP $(\mathrm{cm} / \text { parcela })^{2}$ & Altura $(\mathrm{m} / \text { parcela })^{2}$ & ${\text { Volume }\left(\mathrm{dm}^{3} / \text { parcela }^{2}\right.}^{2}$ \\
\hline EF(\%) & 115,57 & 120,22 & 118,99 \\
Média & 122,8969 & 223,9507 & 107,2942 \\
\hline
\end{tabular}

** Significativo a $1 \%$ de probabilidade pelo teste $\mathrm{F}$. 
gênies. Desta forma, sendo boa a qualidade do experimento, era de se esperar pouca alteração nos resultados entre as diferentes formas de análise.

Os látices são planejados para que cada tratamento ocupe uma quantidade representativa de blocos, mas em geral, no melhoramento, um grande número de progênies é avaliado e uma amostra aleatória de progênies ocupa cada bloco, sendo possível estimar efeitos de bloco com precisão através das informações combinadas das progênies. Desta forma, numa analogia ao látice, pode-se chamar o modelo (c) de recuperação da informação interprogênies.

Quanto às estimativas da variância de progênies, as do modelo fixo (43,7043 para o DAP; 121,7721 para a altura e 176,2026 para o volume) e do modelo do látice $(44,0269$ para o DAP; 126,3080 para a altura e 177,5151 para o volume) mantiveram-se dentro de um intervalo de $95 \%$ de confiança pela aproximação normal para as estimativas pontuais dos modelos (c) e (d). As maiores estimativas pontuais foram, no entanto, consistentemente produzidas quando consideradas como efeitos aleatórios, resultado que está de acordo com Silva (1997). Para o modelo (f), as estimativas foram as que seguem: 44,1478 para o DAP; 136,8919 para a altura e 178,9562 para o volume. Tais estimativas estiveram consistentemente mais próximas das dos modelos (c) e (d), sendo inclusive ligeiramente maiores para a altura. A falta da informação sobre a variância dos blocos dentro de repetições reduz a confiabilidade dessas estimativas, pois seu cálculo depende de operações com componentes ligeiramente inflacionadas, tanto para a variância do erro quanto para a variância de progênies.

A Tabela 2 resume as estimativas dos coeficientes de herdabilidade para os diferentes modelos. Os intervalos de $95 \%$ de confiança (IC) para o parâmetro foram bastante parecidos entre os quatro modelos (a), (b), (c) e (d). Os IC para a análise BLUP (c) e também para o modelo aleatório (d) mostraram-se, no entanto, ligeiramente mais fechados que os demais, indicando serem estas formas, que levam em conta serem as progênies aleatórias, os de melhor estimativa da herdabilidade.

Todas as estimativas pontuais caem dentro de qualquer dos seis IC, o que indica que a alteração na estimativa de herdabilidade é pequena. Deve-se con- siderar que, no caso dos modelos (b), (d) e (f), admitir blocos e repetições como de efeitos aleatórios levaria à inclusão da variância desses efeitos no denominador da herdabilidade. Como esta não é uma prática comum, os efeitos de progênies foram sempre considerados como aleatórios e a situação de seleção será sempre restrita ao ensaio, o que leva a estimativas pontuais bastante próximas, qualquer que seja o método considerado. Uma alternativa seria utilizar o estimador de Singh \& Ceccarelli (1995), para a herdabilidade no sentido amplo, baseado nas estimativas REML de variâncias e covariâncias em blocos incompletos. Neste caso, no entanto, a herdabilidade não guardaria relação com um método de melhoramento. É interessante notar que, devido ao grande tamanho amostral, os IC foram praticamente simétricos em torno da estimativa pontual, o que indica ser a suposição de normalidade na distribuição dos efeitos aleatórios adequada.

As Figs. 1 a 3 mostram as eficiências médias dos ordenamentos para diferentes números de progênies selecionadas, tomando o látice como classificação padrão. Entre os modelos que levam em conta o efeito de blocos, (b) e (d) foram os mais parecidos. A maior semelhança, neste caso, está relacionada a se considerar blocos como efeitos aleatórios. Em todo caso, a diferença entre os ordenamentos (a) e (c) não fica evidente pois ambos estão comparados ao ordenamento (b). O ordenamento do modelo em blocos completos foi o mais discrepante do látice, sendo também muito discrepante do BLUP modelo (c), para todos os caracteres considerados, embora tenha levado a estimativas mais próximas da variância de progênies.

Os resultados indicam que, para uma seleção muito intensa (até $5 \%$ das progênies selecionadas), os modelos podem levar a diferenças de ordenamento relativamente grandes (em média de $10 \%$ a $15 \%$ ); enquanto para intensidades de seleção mais fracas (acima de 15\% em média) os ordenamentos pouco diferem (em média apenas 5\%).

As Figs. 4 a 6 apresentam a variação nos progressos esperados com relação a diferentes números de progênies selecionadas, no tocante aos três caracteres. Se o BLUP for tomado como padrão conceitual de seleção, conforme White \& Hodge (1989), nota-se que os modelos que levam em conta 
o efeito de blocos incompletos são relativamente consistentes na predição do progresso, o mesmo não acontecendo com o modelo de blocos completos.

Os dados parecem indicar que os modelos de estimação diferem mais quanto ao ordenamento para a seleção do que quanto à predição do progresso esperado pela seleção. No entanto, deve-se notar que os resultados do progresso foram calculados segundo cada método, não se tomando um padrão (por exemplo, o progresso na seleção pelo ordenamento do látice medido através do BLUP). Isso faz com que os erros de estimação das componentes

TABELA 2. Estimativas pontuais e por intervalo dos coeficientes de herdabilidade estrito senso (seleção de matrizes com base nas médias de famílias de meios-irmãos) para os diferentes modelos e os três caracteres. Dados em porcentagem. Teste de progênies de $E$. grandis, Salto, 1979, aos sete anos de idade.

\begin{tabular}{|c|c|c|c|c|c|c|c|c|c|}
\hline \multirow[t]{3}{*}{ Modelo } & \multicolumn{9}{|c|}{ Caráter $^{1}$} \\
\hline & \multicolumn{3}{|c|}{ DAP } & \multicolumn{3}{|c|}{ Altura } & \multicolumn{3}{|c|}{ Volume } \\
\hline & LI & Estimativa & LS & LI & Estimativa & LS & LI & Estimativa & LS \\
\hline Fixo & 62,63 & 70,96 & 79,29 & 72,01 & 78,25 & 84,49 & 62,37 & 70,76 & 79,14 \\
\hline Látice & 62,82 & 71,11 & 79,39 & 72,79 & 78,85 & 84,92 & 62,61 & 70,94 & 79,28 \\
\hline BLUP & 62,86 & 71,13 & 79,41 & 74,50 & 80,18 & 85,86 & 62,48 & 70,84 & 79,20 \\
\hline Aleatório & 61,56 & 70,13 & 78,69 & 74,73 & 80,36 & 85,99 & 61,49 & 70,07 & 78,66 \\
\hline$(e)^{2}$ & 62,28 & 70,68 & 79,09 & 72,35 & 78,51 & 84,67 & 61,97 & 70,44 & 78,92 \\
\hline$(f)^{3}$ & 58,50 & 67,75 & 77,00 & 70,12 & 76,78 & 83,44 & 57,52 & 66,99 & 76,45 \\
\hline
\end{tabular}

${ }^{1}$ LI: limite inferior; LS: limite superior.

2 Análise em blocos casualizados com erro efetivo e tratamentos ajustados para blocos, conforme o látice.

3 Análise tomando repetições como blocos completos.

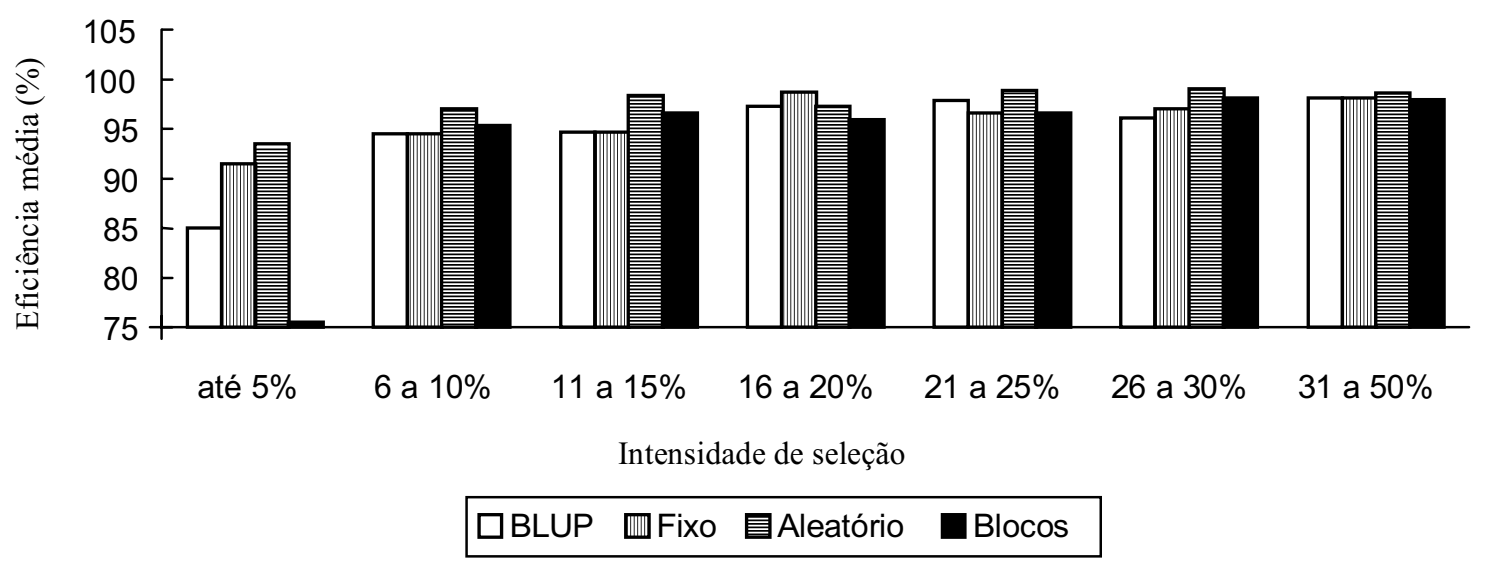

FIG. 1. Eficiência média dos vários modelos em relação à análise como látice, baseada no ordenamento das estimativas (ou predições) de efeitos de tratamentos, considerando-se várias intensidades de seleção para o caráter DAP. 
da variância atenuem as diferenças de ordenamento ao invés de acentuá-las. Em todos os casos, os progressos esperados a partir da análise em blocos completos foram os mais discrepantes podendo ser considerados como estimativas menos robustas.

Os resultados indicaram que a escolha entre a condição de fixos ou aleatórios provoca maiores diferenças nas estimativas no caso de efeitos de blocos do que no de progênies. Isso não deve ser uma tendência geral, e pode ser parcialmente explicado, por ser este um experimento com precisão relativamente alta para a área florestal, o que aumenta a importância da presença ou ausência da recuperação da informação interblocos, para um mesmo número de progênies, mas minimiza as diferenças possíveis (observáveis) entre os ordenamentos.

Os dados não concordam com observações do melhoramento animal, de que para um pequeno nú-

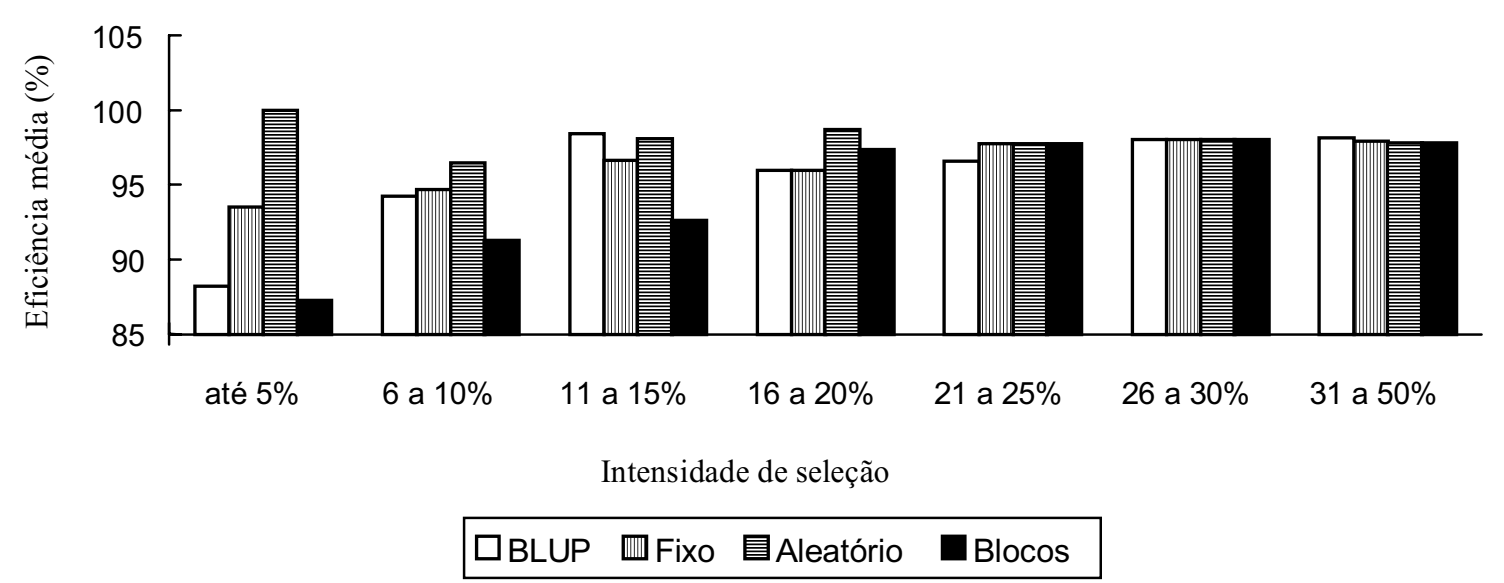

FIG. 2. Eficiência média dos vários modelos em relação à análise como látice, baseada no ordenamento das estimativas (ou predições) de efeitos de tratamentos, considerando-se várias intensidades de seleção para o caráter altura.

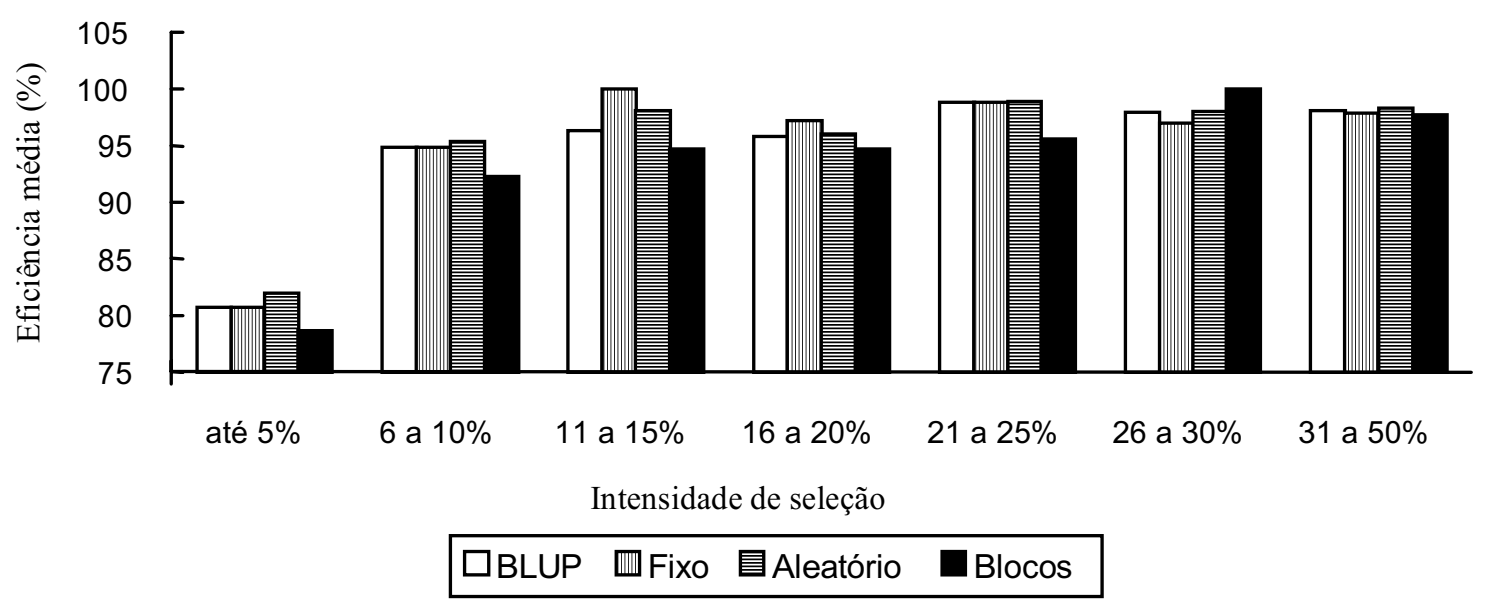

FIG. 3. Eficiência média dos vários modelos em relação à análise como látice, baseada no ordenamento das estimativas (ou predições) de efeitos de tratamentos, considerando-se várias intensidades de seleção para o caráter volume. 
mero de genótipos muito bons o ordenamento não sofre muita alteração, porém, à medida que maior número de genótipos intermediários é considerado, a precisão dos diversos métodos passa a ser da maior importância. Tais resultados, em geral, se aplicam para a seleção entre indivíduos e não entre famílias.

Não se pode julgar pouco importantes as diferenças encontradas entre os métodos de estimação, semelhantes às encontradas por outros autores. Para o melhoramento florestal, pequenas alterações de ordem podem ser importantes especialmente se pensar que as decisões erradas de seleção acumulam-se ao longo das gerações. Além do mais, são diferenças em estimativas a partir dos mesmos dados, indicando a presença de erros que não podem ser ignorados.

Casella \& Berger (1990) definem, entre os fatores da análise da variância, um fator como fixo ou aleatório em termos das generalizações das conclu-

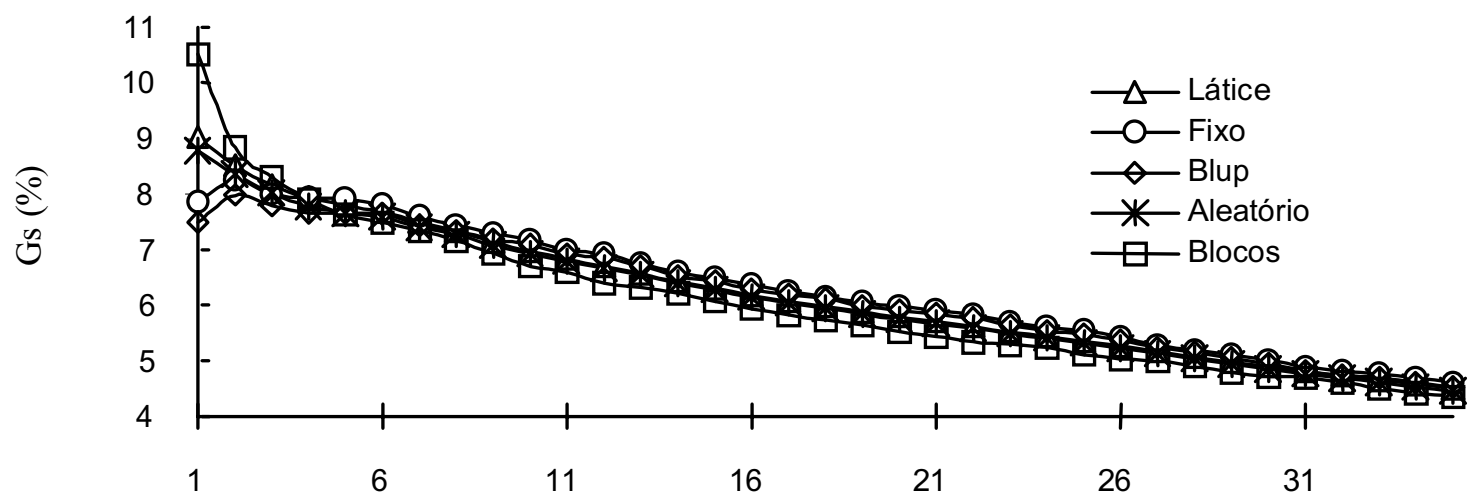

Intensidade de seleção

FIG. 4. Progressos genéticos preditos (Gs\%) para o DAP em função do número de progênies selecionadas de acordo com cada um dos modelos.

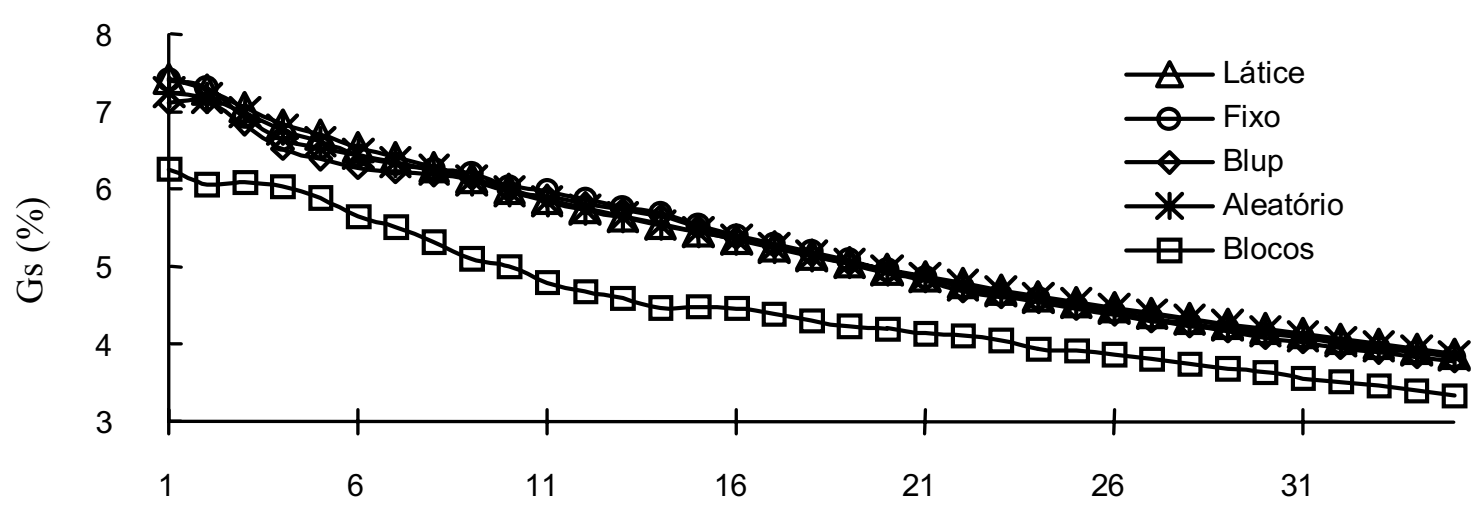

Intensidade de seleção

FIG. 5. Progressos genéticos preditos (Gs\%) para a altura de árvores em função do número de progênies selecionadas de acordo com cada um dos modelos. 


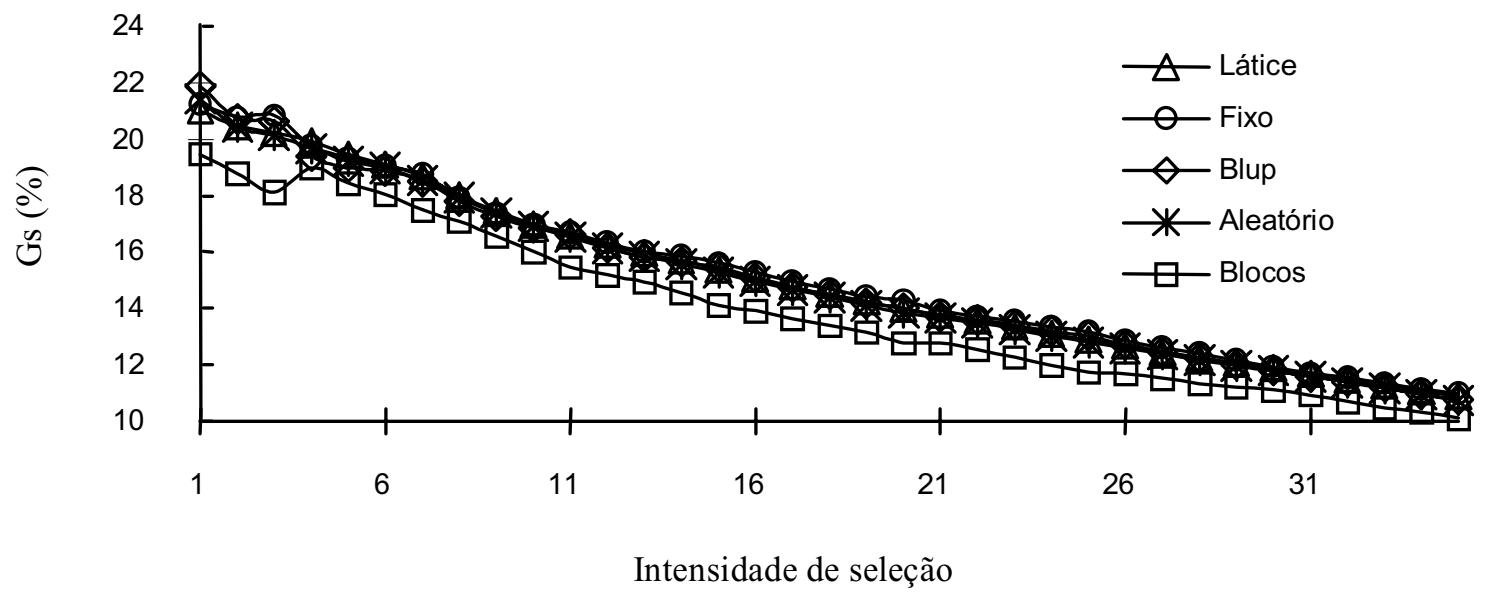

FIG. 6. Progressos genéticos preditos (Gs\%) para o volume em função do número de progênies selecionadas de acordo com cada um dos modelos.

sões, ou seja, o fator é fixo se todos os seus valores de interesse são incluídos no experimento; o fator aleatório é o que não tem todos os seus valores de interesse incluídos no experimento, mas apenas uma amostra aleatória desses valores. White \& Hodge (1989), por sua vez, definem como fixo o fator cujos níveis são efeitos constantes e aleatório o fator cujos níveis estão associados a distribuições de probabilidade.

Pimentel-Gomes (1987) ressalta que a importância da diferença entre a análise intrablocos e a análise com recuperação da informação interblocos é de finalidade: se blocos puderem ser vistos como efeitos aleatórios (e muitas vezes o são na prática agrícola) há a possibilidade de conclusões mais gerais, válidas para toda uma população de blocos.

Desta forma, os efeitos de blocos podem ser considerados tanto como aleatórios ou como fixos, dependendo do objetivo de seleção (se o ensaio for utilizado para a seleção, ou se suas conclusões forem generalizadas para uma população de blocos do ambiente). Isso é o oposto do que se deve dizer das progênies que serão submetidas à seleção: somente podem ser representadas por efeitos aleatórios.

Assim, em termos conceituais o BLUP é a abordagem mais pertinente, podendo no entanto se pensar no modelo aleatório como opção de análise. No primeiro caso, tem-se o ordenamento que mais difere do látice, indicando que pode-se estar incorrendo em considerável erro de seleção pelo emprego da análise usual. Na segunda hipótese, os erros seriam bem menores e possivelmente o ganho com a abordagem de modelos aleatórios para progênies não seria importante.

Para estudos de variabilidade, pode haver vantagem na generalização de expressões de variâncias aditivas e herdabilidades para toda uma gama de locais em que uma população será plantada, sendo então preferível o modelo aleatório. Para fins de seleção, no entanto, é preferível descontar os efeitos fixos diferenciais que se transferem às médias de cada genótipo, impedindo que se reflitam em erros de classificação genética. Neste caso, a forma de análise adequada é o BLUP.

A decisão sobre um ou outro modelo depende de dois tipos de informações, quais sejam: 1- a adequação estatística: neste caso, estudos extensivos de simulação poderiam decidir a real magnitude dos problemas de ordenamento e de estimação de componentes da variância, e 2- a adequação biológica: neste caso, apenas estudos de longo prazo de seleção e ganho realizado poderiam dissipar as dúvidas sobre qual modelo de análise empregar.

\section{CONCLUSÕES}

1. Os delineamentos em látice admitem diferentes modelos de análise; a escolha do modelo tem 
conseqüências importantes tanto na estimativa de componentes da variância e testes de hipótese quanto no ordenamento dos genótipos para fins de seleção; tais diferenças no ordenamento das médias decorrem do método de análise e aumentam à medida que a seleção planejada for mais intensa.

2. A forma de análise interfere mais no ordenamento do que na estimação de parâmetros genéticos e fenotípicos da variabilidade populacional como variâncias e herdabilidades, exceto no caso da análise em blocos completos.

3. Embora o BLUP seja conceitualmente o melhor modelo para seleção, o modelo aleatório também pode ser empregado; neste caso a diferença do modelo usual (látice) é pequena.

4. A definição sobre o melhor padrão de análise em ensaios de progênies (com efeitos fixos ou aleatórios de blocos) depende de uma criteriosa discussão dos objetivos do teste, bem como de estudos mais conclusivos sobre as propriedades estatísticas e biológicas dos modelos.

\section{REFERÊNCIAS}

BUENO FILHO, J.S.S. Modelos mistos na predição de valores genéticos aditivos em testes de progênies florestais. Piracicaba : ESALQ/USP, 1997. 118p. Tese de Doutorado.

BUENO FILHO, J.S.S. Seleção combinada versus seleção seqüencial no melhoramento de populações florestais. Piracicaba : ESALQ/USP, 1992. 96p. Dissertação de Mestrado.

CASELLA, G.; BERGER, R.L. Statistical inference. Belmont : Duxbury, 1990. 650p.

CECON, P.R. Alternativas de análise de experimentos em látice e aplicações no melhoramento vegetal. Piracicaba : ESALQ/USP, 1992. 109p. Tese de Doutorado.

COCHRAN, W.G.; COX, G.M. Diseños experimentales. 3.ed. Mexico : Trillas, 1976.661p.

EBERHART, S.A. Factors affecting efficiencies of breeding methods. African Soils/ Soils Africans, v.15, p.669-679, 1970.

HAMBLIN, J.; ZIMMERMAN, M.J. de O. Breeding common bean for yield mixtures. Plant Breeding Reviews, Connecticut, v.4, p.245-272, 1986.
HENDERSON, C.R. Best linear unbiased estimation and prediction under a selection model. Biometrics, Alexandria, v.31, n.2, p.423-448, 1975.

HENDERSON, C.R.; KEMPTHORNE, O.; SEARLE, S.R.; VON KROSIGK, C.M. The estimation of environmental and genetic trends from records. Biometrics, Alexandria, v.15, p.192-218, 1959

KNAPP, S.J; STROUP, W.W.; ROSS, W.M. Exact confidence intervals for heritability on a progeny mean basis. Crop Science, v.25, p.192-194, 1985.

LATOUR, D.; LATOUR, K; WOLFINGER, R.D. Getting started with PROC MIXED. Cary, NC : Software Salesand Marketing Dep., SAS Institute, 1994. $121 \mathrm{p}$.

MIRANDA FILHO, J.B. Princípios de experimentação e análise estatística. In: PATERNIANI, E.; VIEGAS, G.P. (Eds.). Melhoramento e produção do milho no Brasil. 2.ed. Piracicaba : ESALQ/Campinas : Fundação Cargill, 1987. p.620-650.

PIMENTEL-GOMES, F. Curso de Estatística Experimental. 12.ed. Piracicaba : ESALQ, 1987. 467p.

ROBINSON, G.K. That BLUP is a good thing: the estimation of random effects. Statistical Science, v.6, n.1, p.15-51, 1991.

SAS INSTITUTE. SAS/STAT-Software reference card, release 6.03. Cary, $\mathrm{NC}, 1988.4 \mathrm{p}$.

SILVA, H.D.S. Análise de experimentos em látice quadrado ("square lattice") com ênfase em componentes de variância e aplicações no melhoramento genético vegetal. Viçosa, MG : UFV, 1997. 139p. Dissertação de Mestrado.

SINGH, M.; CECCARELLI, S. Estimation of heritability using variety trials data from incomplete blocks. Theorethical and Applied Genetics, v.90, p.142$145,1995$.

VENCOVSKY, R.; BARRIGA, P. Genética biométrica no fitomelhoramento. Ribeirão Preto : Sociedade Brasileira de Genética, 1992. 496p.

WHITE, T.; HODGE, G. Predicting breeding values with applications in forest tree improvement. Dordrecht : Kluwer Academic, 1989. 367p.

YATES, F. A new method of arranging variety trials involving a large number of varieties. Journal of Agricultural Science, v.26, p.424-445, 1936.

Pesq. agropec. bras., Brasília, v.35, n.2, p.259-269, fev. 2000 